\title{
A Prospective case study on an obese adolescent with obstructive sleep apnea (OSA) before versus One year after bariatric surgery
}

\author{
Basel Habra $^{1 *}$, Jassim Fakhro ${ }^{2}$, Ashraf Soliman ${ }^{1}$ and Abdulla Alhothi ${ }^{1}$ \\ ${ }^{1}$ Department of Pediatric Medicine, Hamad Medical Corporation, Doha, Qatar \\ ${ }^{2}$ Department of General surgery, Hamad Medical Corporation, Doha, Qatar
}

progressive weight gain (Age $=14$ years, wt $=103 \mathrm{Kg}, \mathrm{BMI} 45 \mathrm{~kg} / \mathrm{m} 2 \mathrm{Z}$ score $3.81 \mathrm{SD}$ ), where at this point decision was made to be assessed for bariatric surgery. Patient was admitted to our tertiary surgical center for preoperative optimization and multidisciplinary approach of his medical condition including nighttime CPAP respiratory support, strict diet, family, and patient psychological support. Laparoscopic sleeve gastrostomy (LSG) operation was performed afterword at the age of 14 years. Patient did not have any immediate post-operative complication. He was observed in the PICU for 24 hours and was continued on nighttime CPAP and discharged home within three days on CPAP with close follow up by bariatric surgery team. One-year post-operative follow up, his weight dropped to $57 \mathrm{Kg}$, with BMI 20 (46 kg weight lost, 47.4\%). He had normal glucose tolerance and normal hepatic enzyme level. The one year follow up sleep study showed complete resolution of his OSA. (Figures 1 and 2). He also reported complete resolution of his OSA related symptoms with dramatic improvement of his daily activity, and school performance.

\section{Discussion}

Bariatric surgery has emerged to become the recommended weight loss treatment for severe obesity in adolescents. [4-9] peculiarly favorable come for those with obesity associated comorbidity such as our case $[8,10]$. We have found in our case that bariatric surgery has significantly dropped patient weight, with complete resolution of patient metabolic and OSA complication as documented objectively by follow one year post LSG surgery sleep study.

This case study is one of few reports which have looked objectively at OSA obesity co-morbidity in relation short and long-term post bariatric surgery outcome. Our patient suffered from constellation of symptoms related to his OSA which leads to physical and psychological sequences, this has promoted the indication for bariatric surgery along with his impaired glucose tolerance.

Previous studies has reported resolution of OSA symptoms post bariatric surgery, but off note, these studies did not specify how the diagnosis of OSA was made and no objective sleep study was done to night-time continuous positive airway pressure (CPAP). He underwent ECHO heart which ruled out pulmonary hypertension. Metabolically he had impaired glucose tolerance and very high Homeostatic Model Assessment for Insulin Resistance (HOMA-IR $=22$ ) with $\mathrm{HbA} 1 \mathrm{c}=5.7 \%$, elevated liver enzymes and normal lipogram. Diagnostic sleep study showed moderate OSA (AHI=7.6 AASM score) (Figure 1). He was discharged on CPAP during sleep. During, one year Follow up he complained of episodes of headache and poor school performance and

${ }^{\star}$ Correspondence to: Habra B, MD, Department of Pediatric Medicine, Hamad Medical Corporation, PO Box: 3050, Doha, Qatar, E-mail: bhabra@hamad.qa

Key words: adolescent obesity, bariatric surgery, obstructive sleep apnea

Received: March 29, 2021; Accepted: April 18, 2020; Published: April 21, 2020 


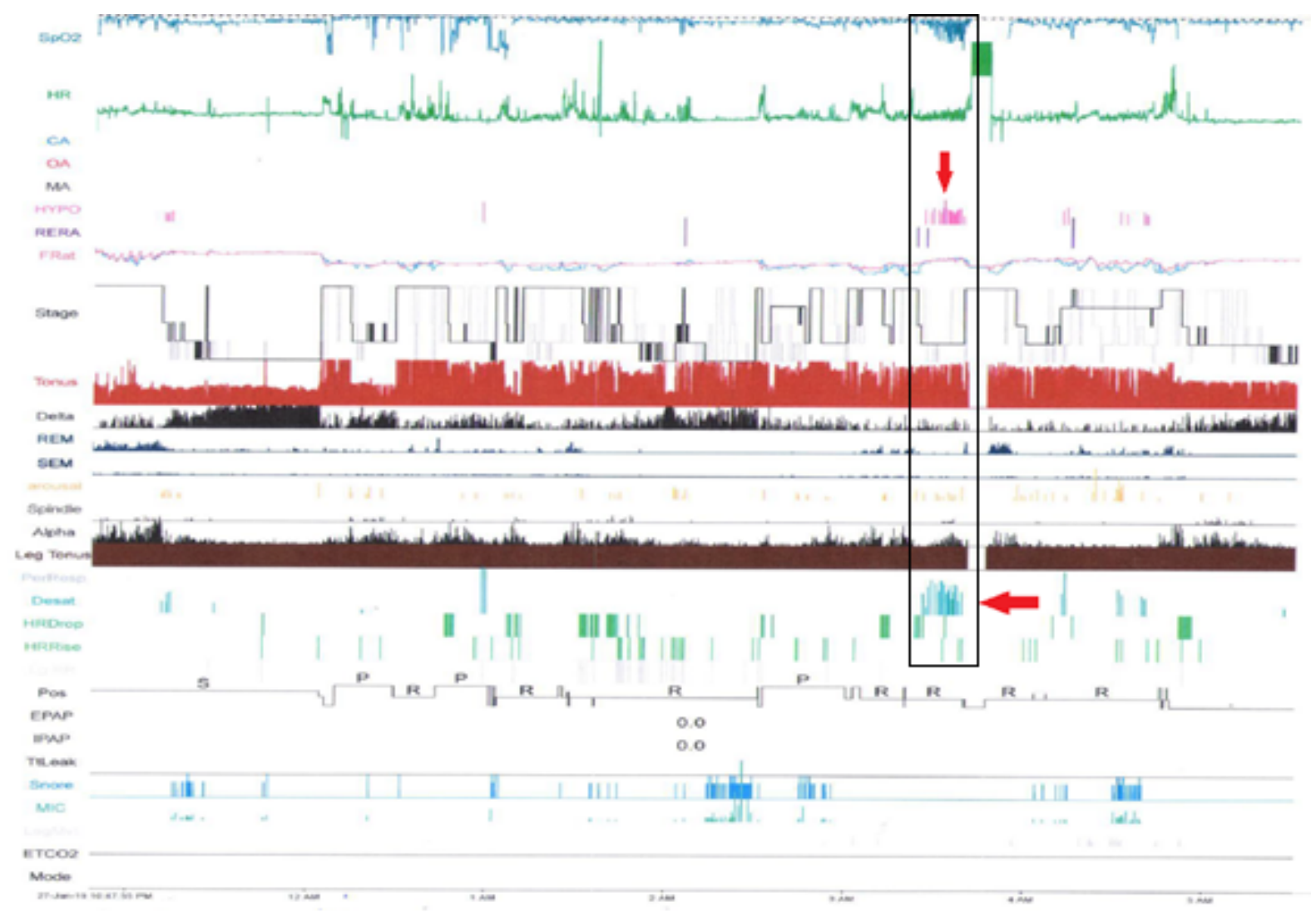

Figure 1. This diagnostic polysomnography for the patient was done off respiratory support and pre-operative which shows episodes of obstructive apneas and hypopnea

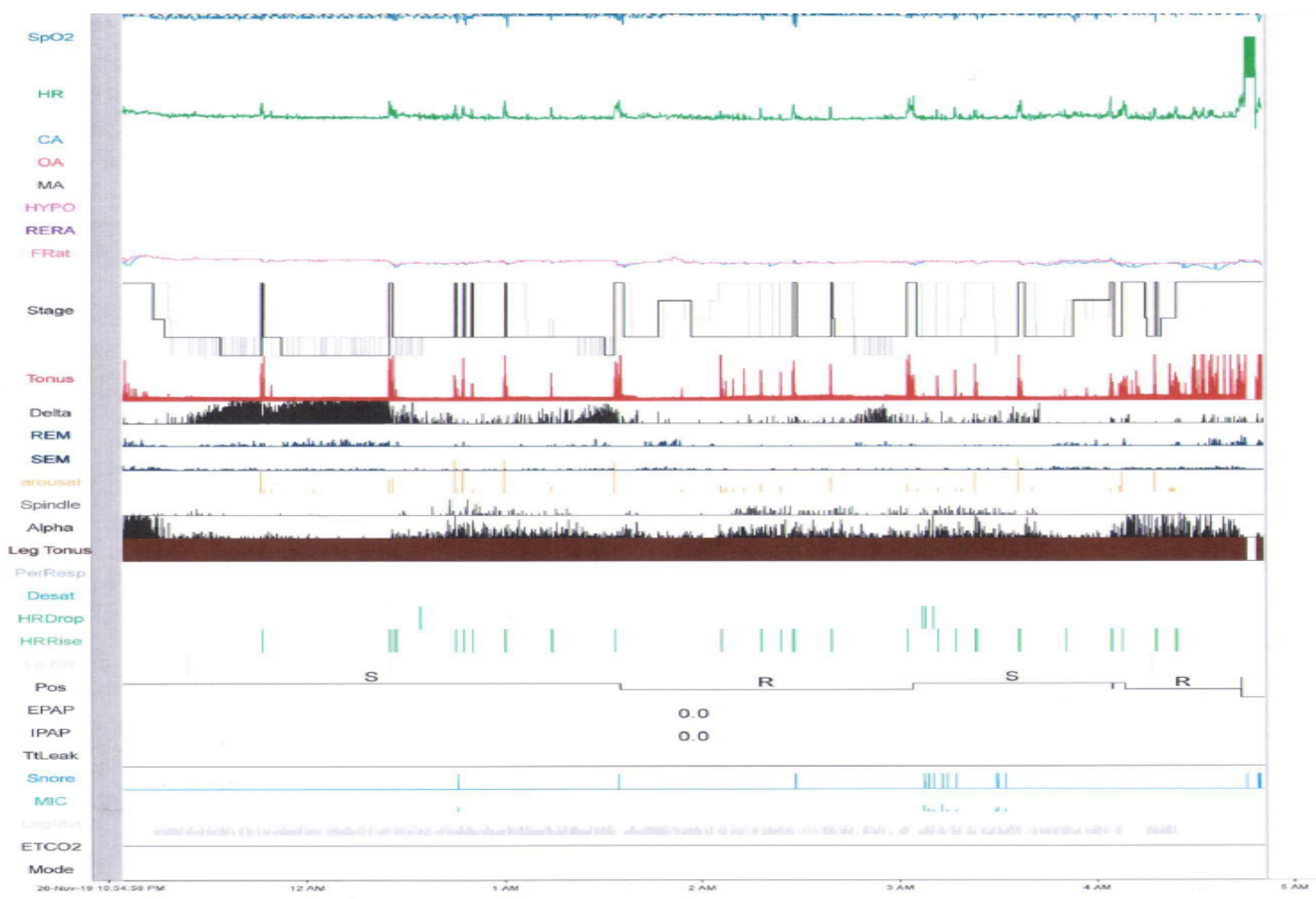

Figure 2. This diagnostic polysomnography for the patient was done off respiratory support and one year post-operative LSG which shows normal sleep architecture with the resolutions of the sleep apnea and hypopnea events 
assess resolution of the OSA post-operatively [10,11]. In our case study the diagnosis of OSA in our patient was confirmed bases on AASM scoring system which consider the gold standard for such diagnosis [12].

Although the mechanisms of OSA in obese adolescent patients is complex are not completely understood, multifactorial elements may contributes to the development this co-morbidity such as the physiological effects of obesity on the respiratory system, upper airway anatomy and lymphoid hypertrophy and possible underlining inflammatory processes [3]. It is unknown the effect of bariatric surgery in such elements but it seems it may play role in clinical reversal of such disease [13].

Our patient did not have any short or long-term complication during 30 days and one year post-operative follow up subsequently, such risk may occur in $8 \%$ of patients [7]. Our patient had lost a significant weight which is slightly higher to the mean drop of weight of what reported previously in other meta-analysis and retrospective studies $[6,9,11]$. Multidisciplinary teams approach and compliance on CPAP for a 6 month post LSG is the key player for such favorable outcomes [10].

Nevertheless, our case study reported objectively the resolution of OSA obesity related co-morbidity in an adolescent patient, this need to be done in a large case controlled population and possible longer duration sleep study follow up to document the sustainable effect of weight lost on such comorbidity.

\section{Conclusions}

This prospective case study showed that bariatric surgery has significantly dropped patient weight, with complete resolution of patient OSA that persisted for one year post-operatively. Multidisciplinary teams approach appeared to be key player for his favorable outcome.

\section{Funding}

This research received no external funding

\section{Conflicts of interest}

The PI, Basel Habra and all associated authors, declare that there is no conflict of interest regarding the publication of this paper.

\section{References}

1. Craig M, Margaret D, Cheryl D, Cynthia L (2017) Prevalence of Obesity Among Adults and Youth: United States, 2015-2016. NCHS Data Brief 288: 1-8. [Crossref]

2. Al-Thani M, Al-Thani A, Alyafei S, Al-Chetachi W, Khalifa SE, et al. (2018) The prevalence and characteristics of overweight and obesity among students in Qatar. The Royal Society for Public Health 160: 143-149. [Crossref]

3. Clodagh M, Giora P (2015) Adolescent Obesity, Adenotonsillar Hypertrophy, and Obstructive Sleep Apnea. Am J Respir Crit Care Med 191: 11. [Crossref]

4. Sarah C, Christopher F, Marc P, Kirk W (2019) Guidance calls for better access to bariatric surgery for teens with severe obesity. AAP 144: 6 .

5. Janey S, Allen B, Nancy T, Matias B, Megan C, et al. (2018) ASMBS pediatric metabolic and bariatric surgery guidelines. Surg Obes Relat Dis 14: 882-901. [Crossref]

6. Felipe E, Federico A, Atsushi E, Hormuzdiyar D, Alessandra Storino, et al. (2017) Weight loss after bariatric surgery in obese adolescents: a systematic review and metaanalysis. Surg Obesity Related Dis 14: 413-422. [Crossref]

7. Inge TH, Thomas I, Meg Z, Todd J, Michael H, et al. (2014) Perioperative Outcome of Adolescents Undergoing Bariatric Surgery: The Teen Longitudinal Assessment of Bariatric Surgery (Teen-LABS) Study. JAMA Pediatr 168: 47-53. [Crossref]

8. Patricia R, Montserrat B, Arturo J (2019) Long-term outcomes of metabolic and bariatric surgery in adolescents with severe obesity with a follow-up of at least 5 years: A systematic review. Surg Obes Relat Dis 15: 133-144. [Crossref]

9. Thomas I, Anita P, Todd M, Marc P, Michael A, Mary L (2016) 374:113-23. Weight Loss and Health Status 3 Years after Bariatric Surgery in Adolescents. N Engl J Med 374: 113-123. [Crossref]

10. Moamena A, Nesreen K, Hussien A, Walid El A, Mohammed AlK (2018) A 5-Year Follow-Up Study of Laparoscopic Sleeve Gastrectomy Among Morbidly Obese Adolescents: Does It Improve Body Image and Prevent and Treat Diabetes? Obes Surg 28: 513-519. [Crossref]

11. Aayed R, Mohamed O, Awadh AlQ (2014) Co-morbidity resolution in morbidly obese children and adolescents undergoing sleeve gastrectomy. Surg Obes Relat Dis 10: 842852 [Crossref]

12. https://aasm.org/clinical-resources/scoring-manual/

13. Michelle L, Kelli-Lee H, Nikhila R, Roberta M (2020) Obstructive sleep apnea in pediatric obesity and the effects of sleeve gastrectomy. Seminars in Pediatr Surg 29: 150887.

Copyright: (C2021 Habra B. This is an open-access article distributed under the terms of the Creative Commons Attribution License, which permits unrestricted use, distribution, and reproduction in any medium, provided the original author and source are credited. 